\title{
Silicon-based three-dimensional microstructures for radiation dosimetry in hadrontherapy
}

Cite as: Appl. Phys. Lett. 107, 023505 (2015); https://doi.org/10.1063/1.4926962

Submitted: 22 April 2015 . Accepted: 23 June 2015 . Published Online: 15 July 2015

C. Guardiola, D. Quirion, G. Pellegrini, C. Fleta (D), S. Esteban, M. A. Cortés-Giraldo, F. Gómez, T. Solberg, A. Carabe, and M. Lozano (iD)
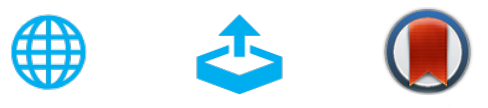

View Online

Export Citation

\section{ARTICLES YOU MAY BE INTERESTED IN}

Spectroscopic properties and radiation damage investigation of a diamond based Schottky diode for ion-beam therapy microdosimetry

Journal of Applied Physics 118, 184503 (2015); https://doi.org/10.1063/1.4935525

Super-thin single crystal diamond membrane radiation detectors

Applied Physics Letters 103, 112106 (2013); https://doi.org/10.1063/1.4821035

The INFN Micro-Si experiment: A silicon microdosimeter for assessing radiation quality of hadrontherapy beams

AIP Conference Proceedings 1530, 148 (2013); https://doi.org/10.1063/1.4812917

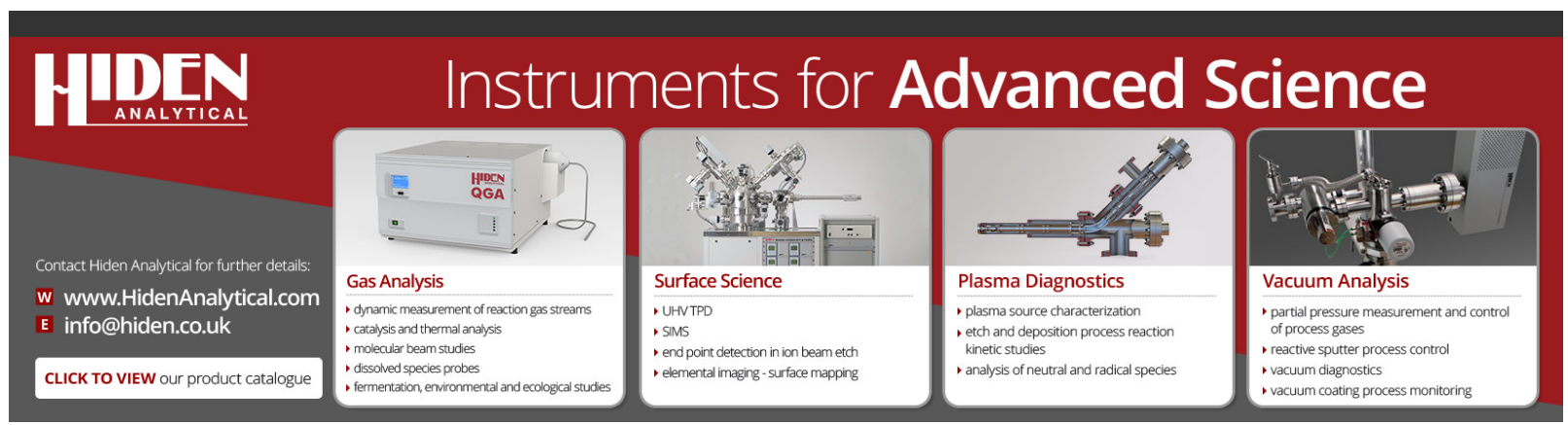




\title{
Silicon-based three-dimensional microstructures for radiation dosimetry in hadrontherapy
}

\author{
C. Guardiola, ${ }^{1, a)}$ D. Quirion, ${ }^{2}$ G. Pellegrini, ${ }^{2}$ C. Fleta, ${ }^{2}$ S. Esteban, ${ }^{2}$ M. A. Cortés-Giraldo, ${ }^{3}$ \\ F. Gómez, ${ }^{4,5}$ T. Solberg, ${ }^{1}$ A. Carabe, ${ }^{1}$ and M. Lozano ${ }^{2}$ \\ ${ }^{1}$ Department of Radiation Oncology, University of Pennsylvania, Philadelphia, Pennsylvania 19104, USA \\ ${ }^{2}$ Centro Nacional de Microelectrónica (IMB-CNM, CSIC), Bellaterra 08193, Spain \\ ${ }^{3}$ Departamento de Física Atómica, Molecular y Nuclear, University of Sevilla, 41080 Sevilla, Spain \\ ${ }^{4}$ Departamento de Física de Partículas, Universidad de Santiago de Compostela, Santiago de Compostela \\ 15782, Spain \\ ${ }^{5}$ Grupo de Imagen Molecular, Instituto de Investigaciones Sanitarias, Santiago de Compostela 15706, Spain
}

(Received 22 April 2015; accepted 23 June 2015; published online 15 July 2015)

\begin{abstract}
In this work, we propose a solid-state-detector for use in radiation microdosimetry. This device improves the performance of existing dosimeters using customized 3D-cylindrical microstructures etched inside silicon. The microdosimeter consists of an array of micro-sensors that have 3Dcylindrical electrodes of $15 \mu \mathrm{m}$ diameter and a depth of $5 \mu \mathrm{m}$ within a silicon membrane, resulting in a well-defined micrometric radiation sensitive volume. These microdetectors have been characterized using an ${ }^{241} \mathrm{Am}$ source to assess their performance as radiation detectors in a high-LET environment. This letter demonstrates the capability of this microdetector to be used to measure dose and LET in hadrontherapy centers for treatment plan verification as part of their patient-specific quality control program. (C) 2015 AIP Publishing LLC. [http://dx.doi.org/10.1063/1.4926962]
\end{abstract}

Radiotherapy (RT) is a type of cancer treatment in which tumors are irradiated with ionizing radiation while limiting the dose to adjacent organs at risk. Delaney ${ }^{1}$ estimated that approximately $52 \%$ of cancer patients receive RT at least once during their treatment. RT has achieved success in a variety of cancers in both a curative and palliative setting (alone or in combination with chemotherapy, surgery, or both). The field has been enhanced with the introduction of advanced techniques such as hadrontherapy, the use of protons and heavier charged particles such as carbon ions. Protons and carbon ions deposit a larger amount of energy per unit particle track length than conventional RT sources, and this is accounted for in terms of radiation quality parameters such as lineal energy $(y)$. The larger the magnitude of this parameter within the particle beam, the more biologically effective the beam is. For this reason, $y$ is one of the required parameters for the radiobiological optimization of proton/carbon treatment plans. The lineal energy is a microdosimetric parameter related to a macroscopic variable called linear energy transfer (LET) that is used for radiobiological optimization. With high-LET particles, the radiobiological effect depends strongly on the magnitude of microdosimetric effects. In this context, the relative biological effectiveness (RBE) is defined as the ratio between the dose required to achieve a given biological effect with conventional RT and the dose required to achieve the same effect with the hadron beam under consideration. $^{2}$ The RBE depends on the type of ion and LET, and since the LET can be much greater for heavy ions, it must be well characterized for hadrontherapy treatment planning. Systems for dose calculation are based on existing RBE models obtained from computational algorithms validated with experimental measurements. ${ }^{3-6}$ Treatment planning

a)Email: GuardioC@uphs.upenn.edu systems (TPS) are used in RT to determine the dose distribution obtained for a certain beam arrangement to be applied to a tumor volume. In the case of hadrontherapy, the treatment planning is challenged by the strong influence of the track structure on the therapeutic effectiveness. A treatment plan which is not optimized for RBE changes along the beam path can lead to both loss of tumor control as well serious long-term side effects due to unintended normal tissue irradiation. Using radiation microsensors that can experimentally verify microdosimetric characteristics would have a fundamental impact on treatment planning for hadrontherapy.

Special considerations must be taken into account when designing a microdosimeter. First, the detector must have a cross-section size on the order of that of a mammalian cellular nucleus (a few micrometers). Second, since the cellular volume may be approximated by a cylindrical shape, the sensitive volume (SV) size of the microsensor must be designed with a well-defined cylindrical volume. ${ }^{8}$ Gas-filled tissue equivalent proportional counters (TEPCs) have traditionally been used for performing microdosimetric measurements. ${ }^{7-9}$ However, TEPCs have several significant disadvantages, including wall effects, high voltage bias, gas supply requirements, and conditions of low irradiation fluence rates. Semiconductor-based radiation detectors that can provide microscopic sensitive volumes can overcome many of these disadvantages. ${ }^{10-19}$ In this letter, we present a radiation detector based on cylindrical 3D-microsensors etched inside the semiconductor bulk. Our microdetector shows some advantages over the last reported silicon microdosimeters. ${ }^{20,21}$ For example, the flat layout on the 3D-microsensor front-face facilitates to figure out the metal strip connection issues, which results in a high functional yield; the well-delimited 3D-cylindrical design avoids the charge-sharing between neighbour pixels, and the field funneling effect is avoided using SOI wafers. 
(A) 3D unit cell

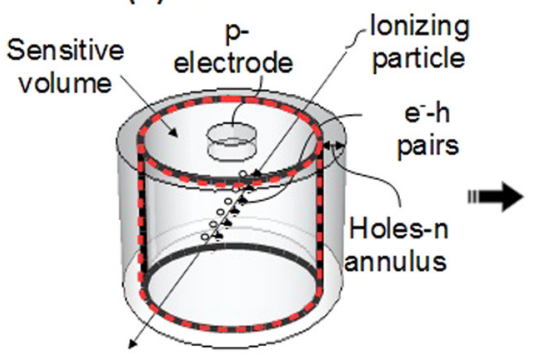

(B) Cross section (2D) unit cell

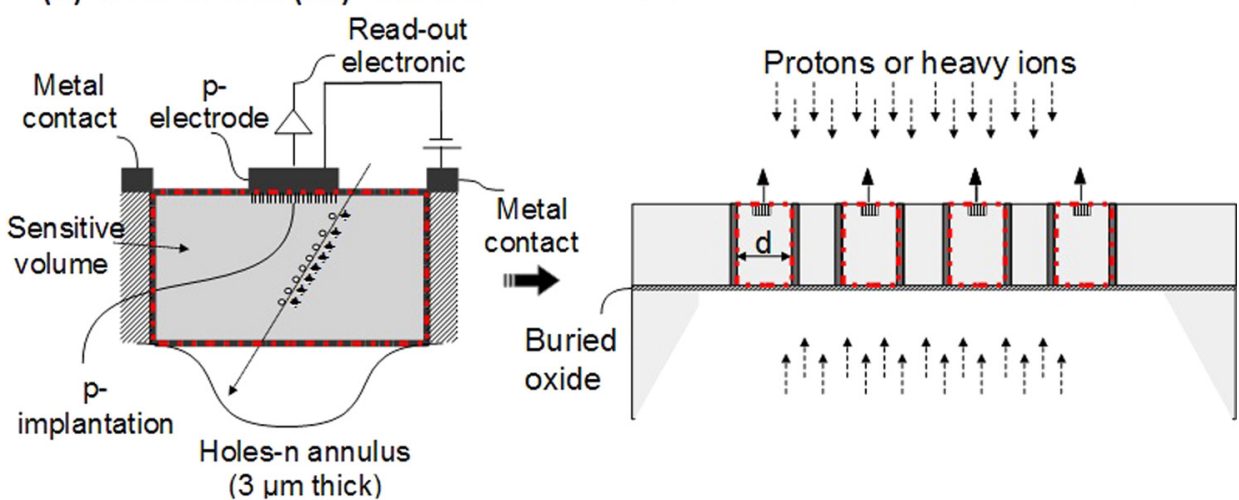

(C) $10 \times 10$ microsensor array

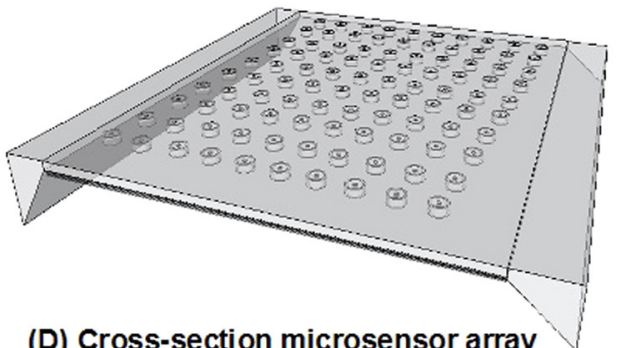

IG. 1. (a) Sketch of a simplified 3Dmicrosensor with a volume equal to the average size of cells to be irradiated. (b) Cross-section of this unit-cell: the electrodes are implanted on the top (p-electrode) and etched as an annulus into the bulk (n-electrode). (c) Sketch of array of $10 \times 10$ microsensors manufactured in a wafer whose support piece is etched. (d) Partial crosssection of the microdosimeter.
Besides, it works with an energy threshold of $4.4 \mathrm{keV} / \mu \mathrm{m}$, the bias is $2 \mathrm{~V}$, the minimum 3D-microsensor pitch is $25 \mu \mathrm{m}$, and it consists of individual pixels (with a customized readout electronic system) that may track lineal energy gradients.

Drawing upon the idea proposed by Parker ${ }^{22}$ for processing columnar electrodes within the semiconductor substrate (instead of being implanted in the surface to manufacture radiation detectors), the Institute of Microelectronics in Barcelona (IMB-CNM) has extended the Parker's 3D-diode concept. ${ }^{23-26}$ Based on this idea, but advancing its initial configuration, we propose a microdosimeter formed by an array of independent 3D-microsensors (Fig. 1) with a well-defined

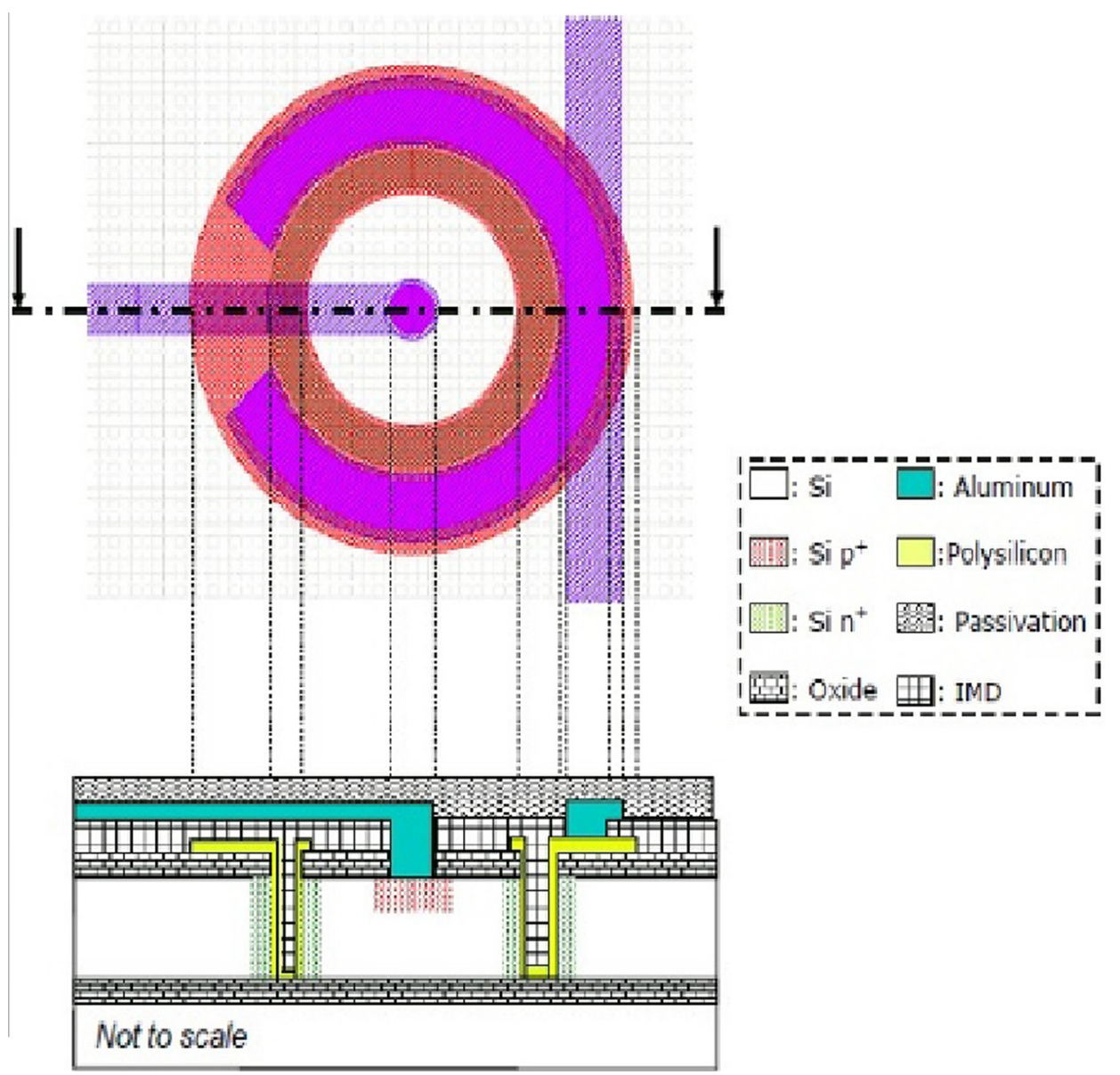

FIG. 2. Cross-section sketch of a 3Dmicrosensor (not to scale). 
TABLE I. 3D-microsensor layers.

\begin{tabular}{lc}
\hline \hline Zone & Layers $(\mu \mathrm{m})$ \\
\hline Active silicon & 5.3 \\
Buried oxide & 1 \\
Field oxide & 0.6 \\
Polysilicon & 0.5 \\
$\mathrm{~N}+$ diffusion & $0.2-0.9$ \\
P+ diffusion & 2.6 \\
Polysilicon/metal dielectric (PMD) & 1.2 \\
Metal & 2 \\
Passivation & $0.2 \mathrm{SiO}_{2}+0.2 \mathrm{Si}_{3} \mathrm{~N}_{4}$ \\
\hline \hline
\end{tabular}

micrometric cylindrical shape etched in the silicon bulk (simulating each cell). ${ }^{27}$

To facilitate this, we have developed a type of diode with a 3D-cylindrical electrode etching (15 $\mu \mathrm{m}$ diameter and $5 \mu \mathrm{m}$ depth) with an inner volume that matches an approximate SV that simulates a cellular or subcellular structure. This microstructure delimits the electric field and avoids the chargesharing amongst adjacent pixels compared with traditional planar detectors in which the electric field pattern causes the charge carriers to drift horizontally far from the pixel boundaries. ${ }^{28}$ Overall, with this design we obtain cell-like silicon $\mathrm{SV}$ s of a few micrometers thick. When a proton or carbon ion passes through the 3D-microsensors, it ionizes the silicon and creates free electron-hole (e-h) pairs that are proportional to the deposited energy by the particles within the 3Dmicrosensor SV. The energy imparted in the silicon SV by the particles, $\varepsilon$, divided by the mean cord length of this SV

$$
\bar{l}=\frac{4 V}{S \times \xi}
$$

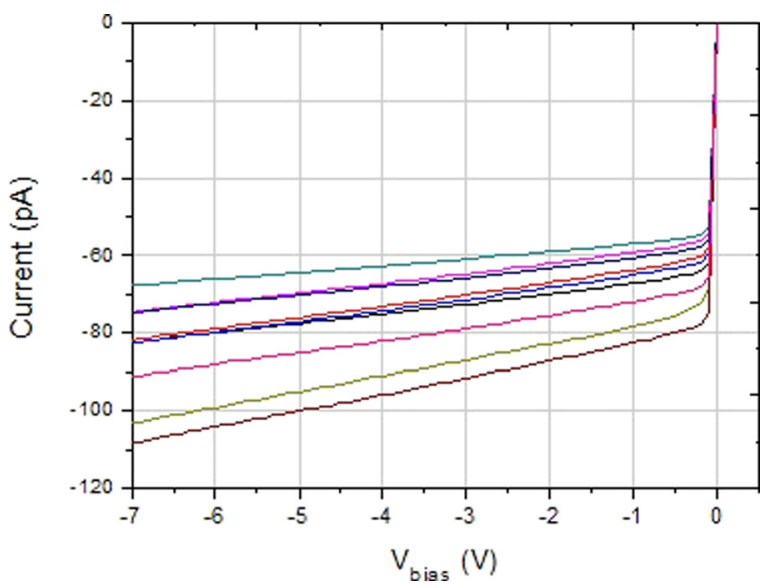

FIG. 4. Current vs bias voltage curves for the characterization of 3Dmicrosensors.

(V and $\mathrm{S}$ are the volume and area irradiated of the SV, and $\xi$ is the tissue-equivalent (TE) conversion factor), and defines the associated lineal energy $(y)^{8}$

$$
y=\frac{\varepsilon}{\bar{l}} .
$$

Depending on the SV geometric shape, the chord length distribution differs significantly, and $l$ and $y$ must then be calculated accordingly. ${ }^{16}$ Lineal energy must be corrected by (i) the charge collection efficiency (CCE) and (ii) the tissue equivalence, i.e., silicon to water. ${ }^{8}$ The microdosimeters are manufactured on 4-in. Silicon-on-Insulator (SOI) wafers with a high resistivity n-type substrate as an array of $11 \times 11$ 3D-microsensors. The device silicon is $\langle 100\rangle$, n-type doped with phosphorus, with a nominal resistivity greater than

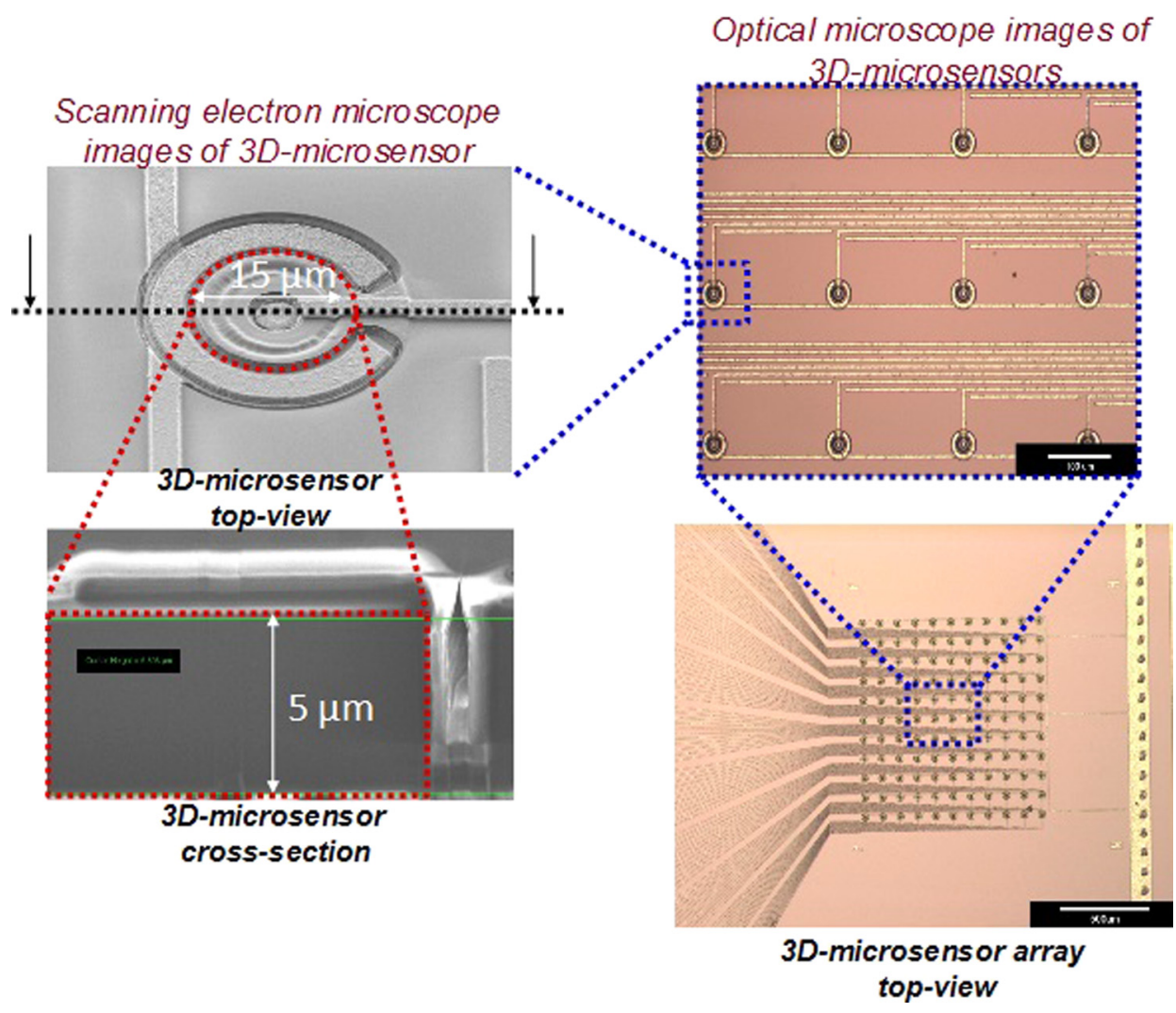

FIG. 3. Left: SEM images of a microsensor $(15 \mu \mathrm{m}$ diameter, $5 \mu \mathrm{m}$ thickness). Right: Optical microscope images of microdosimeter that shows the metal-strips to be connected to an appropriated readout electronics system. 


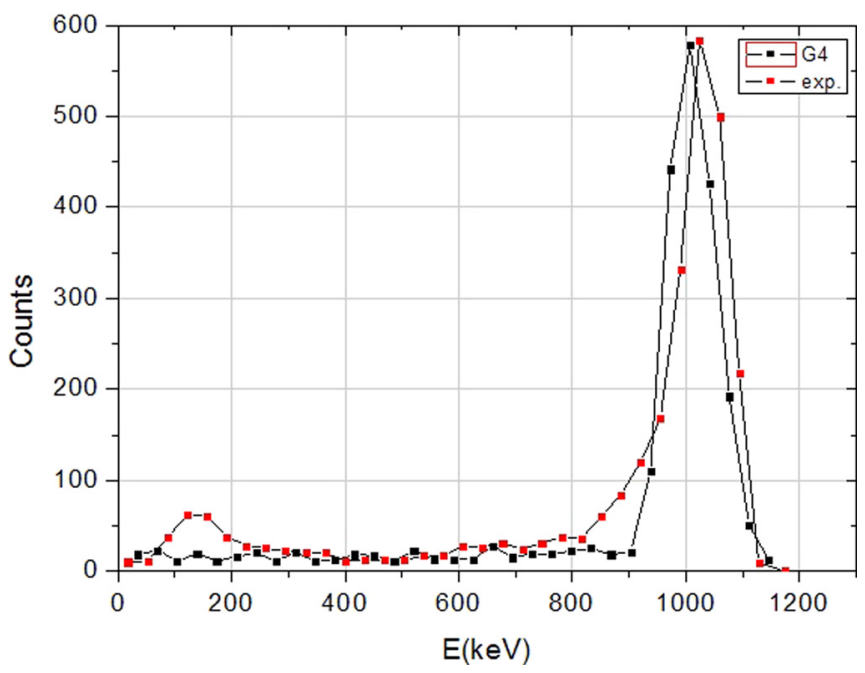

FIG. 5. Measured and Monte Carlo simulated pulse height produced by ${ }^{241} \mathrm{Am}$ alpha source in the 3D-microsensor. The energy threshold is $12 \mathrm{keV}$.

$3 \mathrm{k} \Omega \mathrm{cm}$ and a thickness of $(6 \pm 0.5) \mu \mathrm{m}$. The buried oxide and the support silicon thicknesses are $1 \mu \mathrm{m}$ and $300 \mu \mathrm{m}$ for all the wafers. Figure 2 shows the 3D-microsensor layout where the $p$-and- $n$ electrodes and the metal strips that connect them with the contacts are displayed: the p-type electrodes have a $4 \mu \mathrm{m}$ diameter and are surrounded by a ntype annulus $3 \mu \mathrm{m}$ wide by $5.4 \mu \mathrm{m}$ in deep with various internal diameter $(9,10,15,20$, and $25 \mu \mathrm{m})$ to include a greater number of cell sizes distribution.

Microdosimeters are distributed in a square geometry on the silicon wafer with $25,50,100$, and $200 \mu \mathrm{m}$ pitches (distance between p-columns) and with $9,10,15,20$, and $25 \mu \mathrm{m}$ of internal diameter (D), in order to include a greater number of cell sizes distribution. First, the p-type electrode is defined etching a circular window within the silicon oxide and then an ion implantation with boron $(\mathrm{p}+)$ is made. The annulus is etched using the deep reactive ion etching (DRIE) technique with an Alcatel 601E machine. Next, it is partially filled with $0.5 \mu \mathrm{m}$ polysilicon which is further doped with phosphorus $(\mathrm{n}+)$ to form the $\mathrm{P}-\mathrm{N}$ junction. Estimated depth of the $\mathrm{N}+$ diffusion in silicon is $0.2-0.9 \mu \mathrm{m}$. The top of the holes is metalized with $\mathrm{Al}$ and each electrode is connected with thin Al layers to provide the electrical contact. A dielectric layer is then deposited to fill the trenches and insulate the polysilicon from the aluminum lines which provide the electric contact. Table I summarizes the thickness of each material used to produce 3D-microsensors.

Figure 3 shows both scanning-electron microscope (SEM) and optical images of the 3D-microsensor (left) and a complete microdosimeter, i.e., an array of $11 \times 11$ 3D-microsensors (right). Figure 4 displays the typical diode behavior of current versus bias voltage for some of the individual 3Dmicrosensors shown in Fig. 3. This demonstrates that the devices are fully functional as they can be biased up to reverse voltages higher than full depletion $(<1 \mathrm{~V})$ with leakage current density of $0.5 \times 10^{-4} \mathrm{~A} / \mathrm{cm}^{2}$.

The detectors were connected to a customized readout electronic system ${ }^{29}$ and tested using a ${ }^{241} \mathrm{Am}$ source that emits alpha particles of $5.5 \mathrm{MeV}$. The measurements were carried out in air at a distance of $7 \mathrm{~mm}$ from the detector.

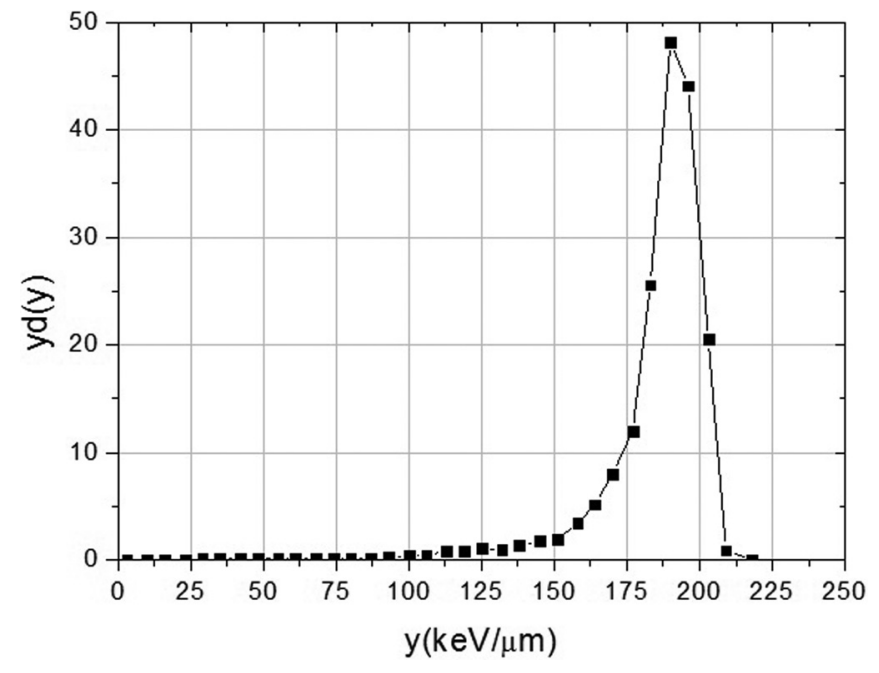

FIG. 6. Measured microdosimetric spectra obtained with an array of 3Dmicrosensors at a distance of $7 \mathrm{~mm}$ from the detector.

Figure 5 shows the simulated (Geant4 Monte Carlo code ${ }^{30}$ ) and measured pulse height distributions of the energy deposited by high-LET alpha particles within a 3D-microsensor of $15 \mu \mathrm{m}$ diameter and $5.4 \mu \mathrm{m}$ thickness, biased at $2 \mathrm{~V}$. It shows very consistent results and demonstrates the feasibility of the 3D-microsensors for the detection of high-LET particles.

Given $f(y)$, the dose distribution $(d(y))$ is expressed as a function of the lineal energy as

$$
d=\frac{y f(y)}{\overline{y_{F}}} .
$$

The mean value of the lineal energy distribution is denoted by the frequency mean lineal energy, $\overline{y_{F}}$. Bearing in mind Eqs. (1) and (2) and the pulse height spectrum obtained with the 3D-microsensors in Fig. 5, the silicon microdosimetric spectra given in terms of the lineal energy is showed in Fig. 6.

In summary, microdosimeters based on 3D-cylindrical microstructures with $5 \mu \mathrm{m}$ thickness and 9, 10, 15, 20, and $25 \mu \mathrm{m}$ internal diameter (and 25, 50, 100, and $200 \mu \mathrm{m}$ pitches) have been fabricated. We have shown feasibility at the level of the average cell size, thus providing a closer measurement of silicon $\Delta \mathrm{E}$. An extended study is currently being carried out in the Roberts Proton Therapy Center at the University of Pennsylvania to characterize the microdosimetric aspects of clinical proton beams. The use of these 3D microdosimeters can enhance the accuracy of RBE calculations normally affected by the inherent uncertainty of Monte Carlo simulations due to the approximation of material composition and energy-dependent physical laws involved in such calculations. The effect of such approximations will be assessed by comparison with absolute measurement of radiation quality parameters with the microdosimeter shown in this letter, which has been specifically customized for hadrontherapy.

Authors thank M. Newcomer and W. Kononenko for allowing us to access the radiation laboratory in UPenn and for helping us with the electronics issues, and D. Lynn for making the wire-bondings of the detectors at BNL. C.G. 
thanks CNM's clean-room staff for the care during the microfabrication process of the microsensors, J. Rodríguez and C. Lacasta for supporting us with the readout-electronics system, and Ramón Areces foundation. C.F. acknowledges the support of the Spanish Ramon y Cajal program. D.Q. thanks JAE-Doc funding. This work was partially financed by the Spanish Ministry of Education and Science through the Particle Physics National Program FPA2013-48308-C22-P.

${ }^{1}$ G. Delaney, S. Jacob, C. Featherstone, and M. Barton, Cancer 104(6), 1129-1137 (2005).

2IAEA 2008, "Relative biological effectiveness in ion beam therapy," IAEA Technical Report Series 461 (IAEA, Vienna, 2008).

${ }^{3}$ M. Beuve, Radiat. Res. 172, 394-402 (2009).

${ }^{4}$ M. Krämer and M. Sholz, Phys. Med. Biol. 45, 3319-3330 (2000).

${ }^{5}$ R. B. Hawkins, Radiat. Res. 160, 61-69 (2003).

${ }^{6}$ Y. Kase, W. Yamashita, N. Matsufuji, K. Takada, T. Sakae, Y. Furusawa, H. Yamashita, and S. Murayam, J. Radiat. Res. 54, 485-493 (2013).

${ }^{7}$ ICRU 1983, "Microdosimetry," ICRU Report 36 (ICRU, Bethesda, MD, 1983).

${ }^{8}$ H. H. Rossi and M. Zaider, Microdosimetry and its Applications (Springer, 1996).

${ }^{9}$ ICRU 1986, "The quality factor in radiation protection," ICRU Report 40 (ICRU, Bethesda, MD, 1986).

${ }^{10}$ A. B. Rosenfeld, P. D. Bradley, I. Cornelius, G. I. Kaplan, B. J. Allen, J. B. Flanz, M. Goitein, A. Van Meerbeeck, J. Schubert, J. Bailey, Y. Takada, A. Maruhashi, and Y. Hayakawa, IEEE Trans. Nucl. Sci. 47(4), 1386 (2000).

${ }^{11}$ H. H. Rossi, IEEE Trans. Nucl. Sci. 23(4), 1417 (1976).

${ }^{12}$ J. F. Dicello, H. I. Amols, M. Zaider, and G. Tripard, Radiat. Res. 82, 441-453 (1980).

${ }^{13}$ M. Orlic, V. Lazarevic, and F. Boreli, Radiat. Prot. Dosim 29, 21 (1989).
${ }^{14}$ A. Kadachi, A. Waheed, M. Al-Eshaikh, and M. Obeid, Nucl. Instrum. Methods Phys. Res., Sect. A 404, 400 (1998).

${ }^{15}$ P. J. McNulty, U.S. patent 5256879 (10 October 1991).

${ }^{16}$ P. D. Bradley, Ph.D. thesis, University of Wollongong, 2000.

${ }^{17}$ P. D. Bradley, A. B. Rosenfeld, and M. Zaider, Nucl. Instrum. Methods Phys. Res., Sect. B 184, 135-157 (2001).

${ }^{18}$ S. Agosteo and A. Pola, Rad. Prot. Dosim. 143(2-4), 409-415 (2011).

${ }^{19}$ S. Agosteo, G. Valvo, P. G. Fallica, and Al. Fazzi, U.S. patent US $8,183,655$ B2 (May 22, 2012).

${ }^{20}$ S. Agosteo, F. Del Corso, A. Fazzi, F. Gonella, M. V. Introini, I. Liippi, M. Lorenzoli, M. Pegoraro, A. Pola, V. Varoli, and P. Zotto, AIP Conf. Proc. 1530, 148 (2013).

${ }^{21}$ J. Livingstone, D. A. Prokopovich, M. L. F. Lerch, M. Petasecca, M. I. Reinhard, H. Yasuda, M. Zaider, J. F. Ziegler, V. L. Pisacane, J. F. Dicello et al., IEEE Trans. Nucl. Sci. 59(6), 3126 (2012).

${ }^{22}$ S. I. Parker, C. J. Kenney, and J. Segal, Nucl. Instrum. Methods Phys. Res., Sect. A 395, 328-343 (1997).

${ }^{23}$ G. Pellegrini, J. Balbuena, D. Bassignana, E. Cabruja, C. Fleta, C. Guardiola, M. Lozano, D. Quirion, and M. Ullán, Nucl. Instrum. Methods Phys. Res., Sect. B 699, 27-30 (2013).

${ }^{24}$ F. García, G. Pellegrini, J. Balbuena, M. Lozano, R. Orava, and M. Ullan, Nucl. Instrum. Methods Phys. Res., Sect. A 607(1), 57-60 (2009).

${ }^{25}$ D. Bassignana, Z. Li, M. Lozano, G. Pellegrini, D. Quirion, and T. Tuuvac, JINST 8, P08014 (2013).

${ }^{26}$ C. Guardiola, C. Fleta, J. Rodríguez, M. Lozano, and F. Gómez, JINST 10, P01008 (2015).

${ }^{27}$ C. Guardiola, F. Gómez, G. Pellegrini, D. Quirion, C. Fleta, and M. Lozano, E.U. patent pending 13/070690 (7 October 2013).

${ }^{28}$ A. MacRaighne, K. Akiba, L. Alianelli, R. Bates, M. van Beuzekom, J. Buytaert, M. Campbell, P. Collins, M. Crossley, R. Dumps et al., JINST 6, P05002 (2011).

${ }^{29}$ R. Marco-Hernández, Nucl. Instrum. Methods Phys. Res., Sect. A 623, 207-209 (2010).

${ }^{30}$ S. Agostinelli, J. Allison, K. Amako, J. Apostolakis, H. Araujo, P. Arce, M. Asai, D. Axen, S. Banerjee, G. Barrand et al., Nucl. Instrum. Methods Phys. Res., Sect. A 506, 250-303 (2003). 